\title{
Common practices in assessing conditions of concrete bridges
}

\author{
MohammedAlsharqawi ${ }^{1, *}$, TarekZayed ${ }^{1}$, and SalehAbu Dabous ${ }^{2}$ \\ ${ }^{1}$ Concordia University, Department of Building, Civil and Environmental Engineering, Montréal, \\ Québec, Canada \\ ${ }^{2}$ University of Sharjah, Department of Civil and Environmental Engineering, Sharjah, United Arab \\ Emirates
}

\begin{abstract}
Bridge condition assessment is an essential step in bridge management. To ensure safety and serviceability of bridge infrastructure, accurate condition assessment is needed to provide basis for bridge Maintenance, Repair, and Replacement (MRR) decisions. In Canada and the United States, visual inspection is the common practice to evaluate a bridge condition. Meanwhile, this practice is limited to detect surface defects and external flaws. For subsurface defects, Non-Destructive Testing and Evaluation (NDT\&E) technologies are being used to supplement visual inspection. This paper reviews the common practices in assessing concrete bridges' conditions and discusses the limitations of available condition assessment models. Further, this research studies six NDT\&E techniques and establishes a set of selection criteria which is utilized to compare each technique in terms of providing the best inspection results. Based on the comparison, it is found that Ground Penetrating Radar (GPR) appears to be the most appropriate NDT\&E techniques for inspection of concrete bridges. Thus, this paper recommends integrating GPR technology with the dominant visual inspection practice in order to establish a more accurate overall bridge condition rating system where surface and subsurface defects are assessed.
\end{abstract}

\section{Introduction}

Deterioration is a foremost problem in any structure during its operation. It occurs due to aging, excessive usage, environmental impacts and other factors. Bridge deterioration results in having more than $26 \%$ of Canada's bridges fall under fair, poor and very poor categories and a value of $\$ 50$ billion is required to replace all of these assets [1]. The aging problem of bridge infrastructure is similar in the US as statistics show that about $28 \%$ of concrete bridge decks is either structurally deficient (SD) or functionally obsolete (FO). ASCE estimated that $\$ 20.5$ billion of annual investment is needed to eliminate the country's bridge deficient backlog by 2028 [2]. The report also graded bridges' conditions as "C + " which refers to mediocre that requires attention. With a large number of aging

*Corresponding author: mohammed.alsharqawi@concordia.ca 
bridges in North America and the growing problem of deterioration across the globe, condition assessment of bridges has been the subject of ongoing research.

\section{Deterioration of concrete bridges}

Large parts of infrastructures were constructed during the 1960's and 1970's. Accordingly, they are facing an increasingly deteriorating problem. Age is one of many factors that decreases infrastructures' robustness. Along with the effect of other factors like external environmental conditions and extreme natural hazards, infrastructure performance is reduced for users [3]. Such factors act as contributors to concrete bridge deterioration and cause vulnerability to any infrastructure. Similar to any reinforced concrete structures, bridges experience loss of integrity over time due to degradation caused by chemical (alkali-silica reaction, carbonation, corrosion, crystallization, leaching, salt and acid action), physical (temperature gradient, fatigue, overloading, shrinkage, freeze-thaw cycles, etc.) and even biological (accumulation of organic matter, living organisms, etc.) mechanisms [4]. It is important to understand different deterioration processes as each process leads to different types of defects such as cracking, scaling, spalling, concrete delamination and corrosion of reinforcing steel. It is also important to identify the basic causes of deterioration which require Maintenance, Repair, and Replacement (MRR) actions; otherwise, ineffective technique may be selected. It is evident that some mechanisms affect the reinforcement and others the concrete itself, yet all degradations mechanisms lead to structure vulnerability and thus reduce the infrastructure reliability.

Corrosion of steel reinforcement, for instance, is considered as the most leading cause of deterioration [5] and has always been viewed as the biggest problem that reasons in many consequent damages as the structure ages. Deterioration comes from the fact that rust, the final product of the electrochemical process of corrosion, occupies much larger volume than the original steel over the years. This causes internal stresses in the surrounding concrete leading to cracking. As corrosion gets more severe, those internal cracks progressively cause partial separation of concrete at the level of reinforcement known as delamination. Several delaminated areas eventually form spalling of concrete which results in structural disintegration. Cracking, delamination, and other discontinuities can occur due to overloading as well. Such defects in some cases could threaten public safety. Examples of cracking, spalling and rebar corrosion are shown in Figure 1.

\section{Condition assessment of bridges}

Bridge structures play a critical role in the transportation system as they serve millions of people on a daily basis. Any failure in these structures will result in both human life and economic loss. Consequently, condition assessment is performed on routine or scheduled basis to ensure public safety and prevent such catastrophic events. Bridge assessment is mainly an interpretation that identifies the appropriate Maintenance, Repair, and Replacement (MRR) activities. With the increasing number of deteriorated bridges in Canada, the US and around the globe, condition assessment techniques of concrete bridges are evolving.

\subsection{Current practices and methods for condition assessment}

Investigation for defects and determination of their severity level are the main objectives of bridge condition assessment. Detailed inspections are conducted regularly in order to discover serious defects and to evaluate the degree of bridge deterioration. Besides, 
emergency or ad hoc inspections due to specific defect are carried out after coincidences or natural disasters such as earthquakes. Concrete bridges are exposed to various forms of deteriorations. Some can be visible on the surface while others are hidden beneath it.
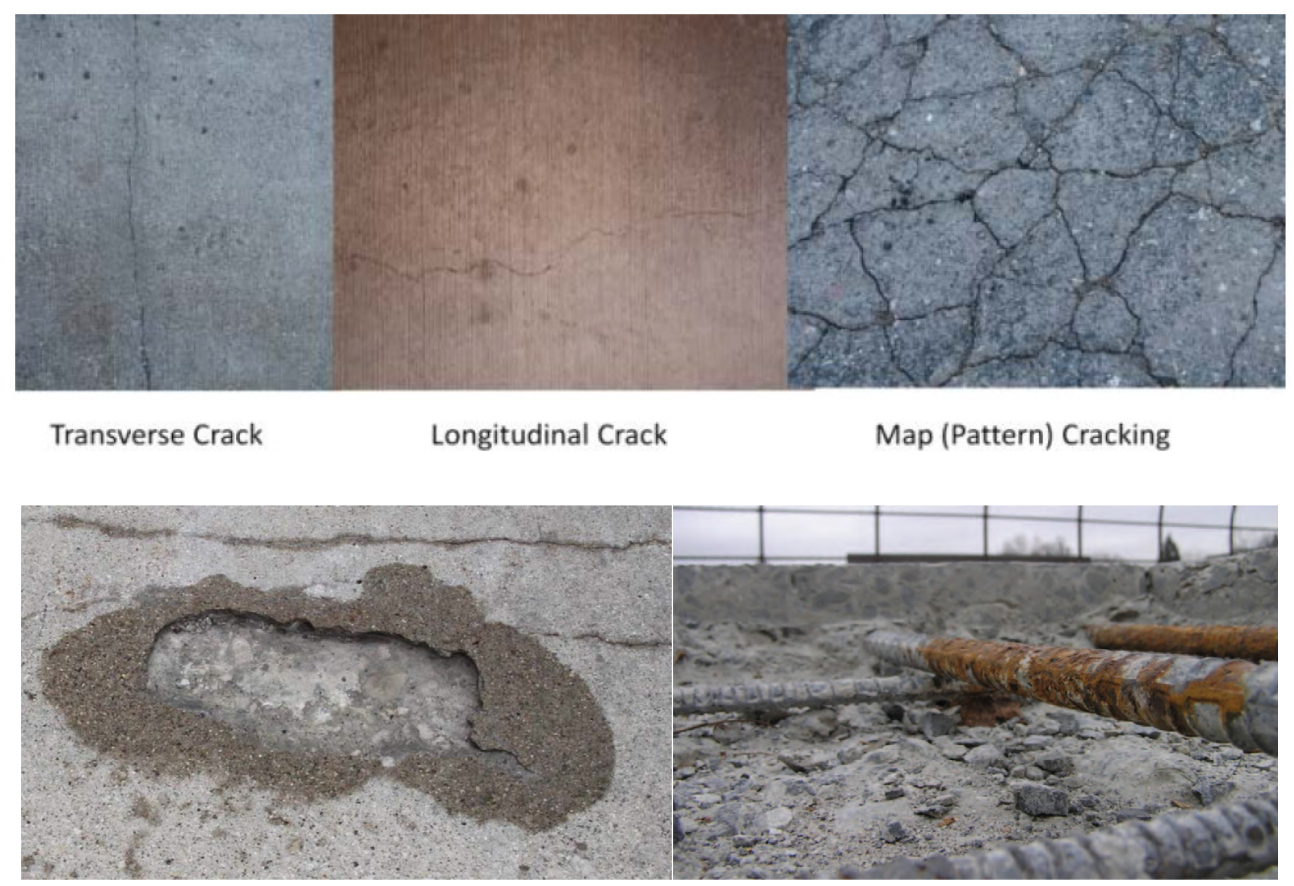

Fig. 1. (a) Classification of concrete cracks (b) Spalling [6](c) Rebar corrosion [7].

In North America, the commonly used techniques to assess bridge conditions is through visual inspection and close observation to bridge elements because it is inexpensive and requires a minimal level of experience. Visual examinations involve using specific techniques to provide valuable information on bridges physical condition. This inspection process can evaluate surface defects such as cracking and spalling. Hammer sounding and chain dragging as shown in Figure 2 are the most commonly used techniques to determine subsurface defects such as delamination where boundaries of delaminated areas within the concrete slab are measured. If severe damages are identified during the visual inspection, a more in-depth condition survey is conducted with the aid of Destructive Testing (DT) and Non-Destructive Testing and Evaluation (NDT\&E) techniques. These two types of techniques are used to assess or inspect the subsurface condition of bridges as reinforcing steel corrosion. The first type is DTs; coring test is the most common test belonging to this class of inspection techniques. DTs provide direct and quick results, but they are expensive, time consuming and have a destructive nature; therefore, they cannot be applied on a regular basis. On the other hand, NDT\&E techniques are less expensive; however, the results are mostly indirect and required some interpretation to get useful outputs.

In order to enhance the current inspection practices, a detailed bridge element inspection system called the "Guide Manual for Bridge Element Inspection" was published in 2011 to introduce an improvement on the widely used Commonly Recognized (CoRe) system of bridge elements. The new guide was built on the concept of element-level condition rating where in-depth assessment of bridge elements is captured. Recently, the Federal Highway Administration (FHWA) has started work on updating the widely used National Bridge Inventory (NBI) coding guide to incorporate the newly developed guide for the detailed 
bridge element inspection and to accurately reflect the condition and performance of highway bridges. In parallel, many transportation agencies supported the adoption and implementation of the detailed element inspection because of its ability to assist in providing sufficient Maintenance, Repair and Replacement (MRR) decisions making and for later analysis in deterioration curves and performance models.

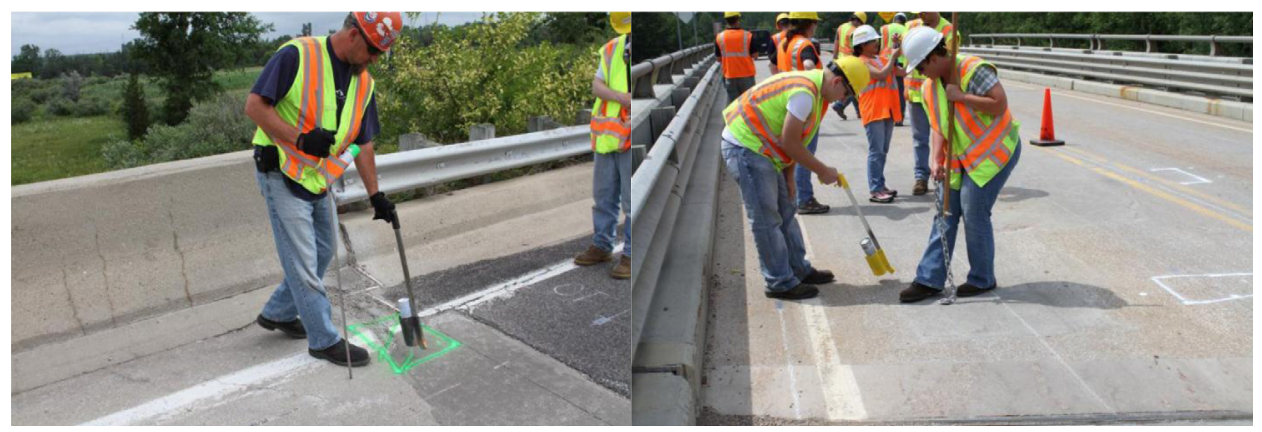

Fig. 2. Hammer sounding and chain-drag testing [6].

\subsection{Previous and related work on condition assessment}

By looking at the literature, previous research works on condition assessment can be categorized into two groups. The first is based on the visual inspection where researchers try to improve the current practices for condition assessment. According to Bolar et al. [5], the condition assessment can be made more effective by determining condition of groups of elements classified based on their resilience. In his paper, he proposed a Hierarchical Evidential Reasoning (HER) framework for the condition assessment of bridge where it is classified into primary, secondary, tertiary and life safety-critical elements. One of the major advantages of using HER is that it has the capability to deal with incomplete and conflicting evidence without making strong assumption about missing data as required in other soft computing methods. Moufti et al. [8] enhanced the application of ER approach by proposing an assessment tool that can handle the prescribed uncertainties in the bridge inspection process and objectively translate the real condition of a bridge through further in-depth measurements. In his model, he hierarchy structured several levels of a concrete bridge under assessment; namely: bridge components, structural elements, and most particularly, the measured defects. Other researchers try to reduce the uncertainties associated with visual inspection and evaluation subjectivity by applying the Fuzzy Set Theory (FST). Several studies on applications of FST with condition assessment of concrete bridges have been reported. Condition evaluation of existing reinforced concrete bridges using fuzzy based analytic hierarchy approach was proposed by Sasmal and Ramanjaneyulu[9]. FST-based evaluation system for existing bridges was proposed by Chen [10] where he emphasis on the use of fuzzy sets to represent bridge condition ratings instead of precise numerical numbers. Jain and Bhattacharjee[11] applied fuzzy concepts to visual assessment of deteriorating specific distress manifestation in reinforced concrete structures. Some of the drawbacks of the above mentioned approaches are: i) the provided condition assessment depends mainly on visual inspection which can be imprecise ii) weights of bridge elements are measured by simple techniques such as Analytical Hierarchy Process (AHP) without considering interdependency and iii) some of the studies consider specific deterioration mechanisms and certain type of defects.

The other research group utilizes technologies for condition assessment. Advanced technologies have been utilized to provide information about deteriorations of bridge 
elements. For surface deteriorations such as cracking, a digital image processing was developed by Adhikari et al. [12] for crack quantification in such a way that it mimics the on-site visual inspections. Such developments will resolve the subjectivity problem, but still it is limited to certain type of defects. For subsurface deteriorations, a number of NonDestructive Testing and Evaluation (NDT\&E) technologies are used to evaluate subsurface conditions on bridge decks. For instance, Washer et al. [13] used Infrared thermography (IR), IR mostly used to detect voids and delamination in concrete bridge decks through measuring the radiant energy emitted from the surface. Gucunski et al. [4] used Ultrasonic Pulse-Echo (UPE) and Half-Cell Potential (HCP). UPE technology uses ultrasonic (acoustic) stress waves assess in detecting defects in concrete elements, debonding of reinforcement bars, shallow cracking, and delamination. HCP measures the potential corrosion of steel reinforcement and prestressed concrete structures. Chase [14] used Impact Echo (IE), IE detects and characterizes delamination within concrete bridge decks. Tarussov et al. [15] used Ground Penetrating Radar (GPR), GPR utilizes electromagnetic (EM) waves that have the ability to identify the subsurface defects locations for both corrosion of steel and also some concrete delamination. Table 1, summarizes the main limitations of the above mentioned technologies. In general, the main disadvantages of using NDT\&E technologies are relatively the high cost and complex data interpretation in addition to detecting certain type of defects.

Table 1. Main NDT\&E technologies limitations[16].

\begin{tabular}{|c|l|}
\hline NDT\&E Technology & \multicolumn{1}{|c|}{ Main Potential Limitations } \\
\hline Infrared thermography (IR) & -Mostly detect voids and delamination in concrete \\
\hline Ultrasonic Pulse-Echo (UPE) & $\begin{array}{l}\text {-Detect debonding of reinforcement, shallow cracking, and } \\
\text { delamination }\end{array}$ \\
\hline Half-Cell Potential (HCP) & $\begin{array}{l}\text {-Evaluate the probable steel corrosion activity in concrete } \\
\text { structures }\end{array}$ \\
\hline Impact Echo (IE) & -Detect only delamination within bridge decks \\
\hline Ground Penetrating Radar (GPR) & -Limited to steel corrosion and potential delamination \\
\hline
\end{tabular}

\subsection{Comparison and selection of NDT\&E technique}

There are many NDT\&E technologies that can be utilized for reinforced concrete bridge inspection. In this task, the available NDT\&E technologies were studied and analysed to highlight the detection capabilities as well as investigate their limitations. The examined techniques include: Infrared Thermography, Ultrasonic, Half-Cell Potential, Impact Echo, Ground Penetrating Radar and Chain drag is added as a classical NDT\&E technique. Theoretically, some or all of the techniques can be performed on the same bridge. Obviously, the bridge condition, in that case, would be better assessed as each technique will eliminate the drawbacks of the others. That solution may exist but would require more time and financial resources to inspect and interpret the results than the case if one single method is selected. In a research sponsored by the Federal Highway Administration (FHWA) in cooperation with the American Association of State Highway and Transportation Officials (AASHTO), Gucunski et al. [7] performed an independent evaluation of the capabilities and limitations of the most common NDT\&E techniques to detect and characterize typical deterioration mechanisms in concrete bridge decks. Based on several performance criteria as well as field and laboratory testings, it was reported that 
among tested NDT\&E technologies, GPR ranks first for condition assessment of concrete bridge decks.

Being aware of the fact that the best selection or combination of NDT\&E technologies should be determined based on full cost-benefit analysis, however due to the time limitation, it was determined in this study that only one NDT\&E technique is employed and only technical benefits are considered for its selection. These technical benefits, or selection criteria, have been identified including: (1) capable of detecting corrosion-induced defects such as (1a) corrosion and (1b) delamination; (2) does not require traffic close for bridge inspection; (3) inspection result can be reproducible; (4) usable for various bridge elements; (5) works well with asphalt overlays that is commonplace in Canada; (6) objective with minimal subjective interpretation from operators; and (7) can be used as a stand-alone technique that does not require other tests.

As shown in Table 2, consistent with the result of Gucunski et al. [7], Ground Penetrating Radar (GPR) also appears to be the most appropriate technology.

Table 2. Comparison of NDT\&E techniques to selection criteria.

\begin{tabular}{|c|c|c|c|c|c|c|c|c|}
\hline \multirow[t]{2}{*}{ NDT\&E Techniques } & \multicolumn{8}{|c|}{$\begin{array}{l}\text { Selection Criteria } \\
\text { (1a): capable of detecting corrosion } \\
\text { (1b): capable of detecting delamination } \\
\text { (2) : does not require traffic close } \\
\text { (3) : inspection result can be reproducible } \\
\text { (4) : usable for various bridge elements } \\
\text { (5) : works well with asphalt overlays } \\
\text { (6) : objective with minimal subjective interpretation } \\
\text { (7) : can be used as a stand-alone technique }\end{array}$} \\
\hline & (1a) & (1b) & (2) & (3) & (4) & (5) & (6) & (7) \\
\hline Infrared Thermography & & $\mathrm{x}$ & $\mathrm{x}$ & & & & $\mathrm{x}$ & $\mathrm{x}$ \\
\hline Ultrasonic & & & & $\mathrm{x}$ & $\mathrm{x}$ & & $\mathrm{x}$ & $\mathrm{x}$ \\
\hline Half-Cell Potential & $\mathrm{x}$ & & & $\mathrm{x}$ & $\mathrm{x}$ & & $\mathrm{x}$ & $\mathrm{x}$ \\
\hline Impact Echo & & $\mathrm{x}$ & & $\mathrm{x}$ & $\mathrm{x}$ & $\mathrm{x}$ & $\mathrm{x}$ & $\mathrm{x}$ \\
\hline $\begin{array}{c}\text { Ground Penetrating } \\
\text { Radar } \\
\end{array}$ & $\mathrm{x}$ & $\mathrm{x}$ & $\mathrm{x}$ & $\mathrm{x}$ & $\mathrm{x}$ & $\mathrm{x}$ & $\mathrm{x}$ & $\mathrm{x}$ \\
\hline Chain Drag & & $\mathrm{x}$ & & & $\mathrm{x}$ & & & $\mathrm{x}$ \\
\hline
\end{tabular}

\section{Summary and conclusion}

It has been observed from the current practices that the commonly used techniques to assess bridge conditions are mainly through visual inspection to bridge elements. Inaccurate measurements and subjective judgment are considered as a drawback of this approach. In order to improve that inspection process, many studies have been executed to account for this uncertainty and subjectivity. However, this practice is still limited to detect surface defects and external flaws. Subsurface defects are mostly measured with the aid of NDT\&E techniques. Moreover, NDT\&E technologies are involved in objectifying the inspection process and making it more reliable. These technologies are promising in terms of providing improvements to the traditional inspection processes; however, it is expensive and limited to certain type of defects. In this study, a selection criteria has been identified considering technical benefits of six NDT\&E techniques. It is concluded that Ground Penetrating Radar (GPR) appears to be the most appropriate technology in terms of providing the best inspection results. Moreover, a current research is in progress to develop a model that integrates the dominant visual inspection practice with the selected NDT\&E 
technique. Hence, it is expected from this research to enhance the condition assessment of bridges by considering both surface and subsurface defects and thus establishing a more accurate condition rating.

\section{References}

1. CIRC, Report card for Canada's infrastructure (2016)

2. ASCE, Report card for America's infrastructure (2013)

3. R. Zimmerman, C. E. Restrepo, J. S. Simonoff, The age of infrastructure in a time of security and natural hazards (2009)

4. N. Gucunski, F. Romero, S. Kruschwitz, R. Feldmann, A. Abu-Hawash, M. Dunn, TRR Journal. 2201, 34-44 (2010)

5. A. Bolar, S. Tesfamariam, R. Sadiq, StructInfrastruct E.9, 648-666 (2013)

6. R. Hoensheid, Evaluation of Surface Defect Detection in Reinforced Concrete Bridge Decks using Terrestrial LiDAR (2012)

7. N. Gucunski, A. Imani, F. Romero, Non-destructive testing to identify concrete bridge deck deterioration (2013)

8. S. Moufti, T. Zayed, S. Abu Dabous, TRR Journal. 2431, 88-96 (2014)

9. S. Sasmal, K. Ramanjaneyulu, Expert Syst Appl.35, 1430-1443 (2008)

10. M. Chen, $C A R^{\prime} 09$, 269-272 (2009)

11. K. K. Jain, B. Bhattacharjee, J ConstrEngManag. 138, 399-408 (2011)

12. R. Adhikari, O. Moselhi, A. Bagchi, Automat Constr.39, 80-194 (2014)

13. G. Washer, N. Bolleni and R. FenwickTRR Journal. 2201, 27-33 (2010)

14. S. Chase, Y. Adu-Gyamfi, P. Tunuguntla, Transportation Research Board 94th Annual Meeting, 15 (2015)

15. A. Tarussov, M. Vandry, A. De La Haza, Constr Build Mater.38, 1246-1254 (2013)

16. M. Alsharqawi, S. Abu Dabous, T. Zayed, Transportation Research Board 95th Annual Meeting, 16-4431 (2016) 